\title{
Harnessing the CD8+ T-cell subsets with stemness for tumor immunotherapy
}

\author{
Wei Wang ${ }^{1,2}$, Yukai He ${ }^{\ddagger, 3}$ \& Sha Wu W $^{*} \ddagger 4$ \\ ${ }^{1}$ Department of Obstetrics \& Gynecology, The First Affiliated Hospital of Guangzhou Medical University, Guangzhou, Guangdong, \\ PR China \\ 2Department of Obstetrics \& Gynecology, Nanfang Hospital, Southern Medical University, Guangzhou, Guangdong, PR China \\ ${ }^{3}$ Georgia Cancer Center, Department of Medicine, Medical College of Georgia, Augusta University, Augusta, GA, USA \\ ${ }^{4}$ Department of Immunology, School of Basic Medical Sciences, Southern Medical University, Guangdong Provincial Key Laboratory \\ of Proteomics, Guangzhou, Guangdong, PR China \\ *Author for correspondence: shawumail@yahoo.com \\ ¥Authors contributed equally
}

' In this short review, we summarize current approaches that enable antigen-specific T cells to persist in vivo to achieve enhanced antitumor effects."

First draft submitted: 25 March 2018; Accepted for publication: 14 June 2018; Published online: 24 August 2018

Keywords: CD8+ T cells • stemness • tumor immunotherapy

A significant number of studies show that the host immune system plays an important role in the fight against malignancy. Naive CD8+ T cells are activated, expanded and differentiated into effector cytotoxic T cells (CTLs) and memory $\mathrm{T}$ cells after optimal stimulation through T-cell receptor (TCR)-pMHC (peptide MHC complex) engagement (signal 1), CD28-B7 co-stimulation (signal 2) and provision of proper cytokines (signal 3). Alternatively, using the genetic engineering approach, a patient's own T cells can be reprogrammed with TCR or chimeric antigen receptor (CAR) genes to create tumor antigen-specific TCR-T and CAR-T cells. At the effector phase, the vaccineactivated CTLs or the genetically engineered TCR-T and CAR-T cells are able to recognize and kill target tumor cells. Unfortunately, these immune effector $\mathrm{T}$ cells, upon entering into the tumor microenvironment (TME), not only meet with a variety of immune suppressive cells and factors that limit their functions, but also are under strong and persistent antigen stimulation that often drive them to exhaustion and apoptosis [1]. Thus, in addition to counteracting the immune suppression mechanisms in TME, generation of antigen-specific T-cell subsets that are capable of robust proliferation and survival in the presence of persistent tumor antigen stimulation in TME will likely generate more potent antitumor effects. In this short review, we summarize current approaches that enable antigen-specific $\mathrm{T}$ cells to persist in vivo to achieve enhanced antitumor effects.

\section{Stem-like memory T cells for better proliferation \& survival}

According to the current models [1,2], naive T cells, after antigen stimulation, will undergo activation, proliferation and stepwise differentiation into stem-like memory $\mathrm{T}$ cells $(\mathrm{Ts} \mathrm{cm})$, central memory $\mathrm{T}$ cells $(\mathrm{T} \mathrm{cm})$, effector memory $\mathrm{T}$ cells (Tem) and effector T cells (Teff). Tscm are at an early differentiation stage and have the stem-like profile.

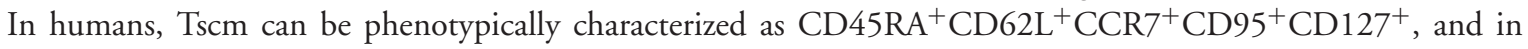
mice the $\mathrm{ScaI}^{+} \mathrm{CD} 44^{-} \mathrm{CD} 62 \mathrm{~L}^{+}$Tscm cells were found to be responsible for chronic HIV infection and graftversus-host disease [3]. Tscm is endowed with self-renewing ability, enhanced proliferative potential, longevity and immune-reconstitution to differentiate into multiple spectrum of other $\mathrm{T}$ cells, and thus may overcome the TME immunosuppressive obstacles and generate persistent antitumor effects. Thus, different strategies have been explored to generate $\mathrm{T}$ cells with stemness [2].

\section{Strength of signal 1 TCR signaling}

The strength of TCR signaling drives a multitude of T-cell functions from proliferation to differentiation. A strong TCR signaling is required to start the process of T-cell activation and differentiation. However, persistent strong

Future Medicine 
TCR signaling may drive the T cells to become terminal, Teffs are prone to be apoptotic. In contrast, a weak TCR signaling may not be capable of activating $\mathrm{T}$ cells properly, but will halt T-cell differentiation at the Tscm stage during immune priming. We recently found that high affinity TCR-pMHC engagement will initiate T-cell activation and differentiation. However, for some T cells, their TCR level is immediately downregulated after initial activation, which weakens the TCR signaling strength, resulting in more Tscm in the antigen-activated T-cell population [4]. The Tscm generated because of TCR downregulation had better antigen responsiveness when re-encountering cognate antigen. We also found that the weak TCR signaling also protects CTLs from exhaustion and apoptosis in tumor lesion and could survive persistent antigen stimulation and thus generate stronger antitumor effects. This high-affinity TCR-pMHC engagement and rapid downregulation may be critical for generating Tscm and for achieving enhanced antitumor effects. Presotto's lab found optimal-affinity TCR signaling endows CD8+ T cells to potent proliferative ability with SHP-1-ERK1/2 and SHP-2-MAPK pathway, but high affinity of TCRs only results in poor and transient MAPK activation and weak proliferation. [5].

\section{Co-stimulatory pathway (signal 2) \& Tscm generation}

In addition to the $\mathrm{CD} 3 \zeta$ chain, the co-stimulatory signaling pathways of CD28, OX40, ICOS, 4-1BB and so forth, have been used as part of the intracellular signaling domain (ICD) to create CAR-T cells. And, different intracellular signaling domain may endow T cells different capability of proliferation and survival [6]. The common membrane proximal YMNM and YXXM motifs of CD28 and ICOS induce activation of NFAT, AP-1 and NF-кB family transcription factors to promote IL-2 synthesis and GSK-3 $\beta$ dephosphorylation, mediate the longevity and survival of CD8 $+\mathrm{T}$ cells. As an activation-induced costimulatory molecule, 4-1 BB has been found potential induce stem-memory CD8 $+\mathrm{T}$ cells formation. The downstream of 4-1BB involves the TRAF1/3-ASK1-JNK-MAPK and TRAF1-MEKK-ERK1/2-NF- $\mathrm{B}$ cascades, contributes to production of IL-2 and IFN- $\gamma$ and upregulation of the antiapoptotic Bcl-2 family members Bcl-xL and Bfl-1 in CD8+ T cells. Several studies suggest that the CD28 intracellular domain stimulates greater CAR-T-cell functionality, whereas the 4-1BB intracellular domain promotes greater CAR-T-cell persistence [7]. The 4-1BB structure is used to construct into the TCR-engineered system to prolong the longevity of CAR-T, such as CD19-CAR-T cells in patients with B-cell malignancies (NCT03019055).

\section{Signaling 3 pathway \& Tscm generation}

The cytokines with the common $\gamma$ chain ( $\gamma$ c-CD132) family such as IL-2, IL-7, IL-15 and IL-21, significantly, induce Tscm differentiation, and the downstream of cytokines signaling involves JAK1/3, STAT3/5, followed by the PI3K/AKT/mTOR and Ras/Raf/MAPK-ERK cascades and the consequently increased activation of c-fos/jun, c-Myc, NF- $\mathrm{kB}$ and Bcl-2 and the decreased expression of PUMA and Bim promote CD8+ T-cell proliferation and survival [8]. These cytokines effectively drive CD8+ $\mathrm{T}$ cells into stem-like phenotypes in vitro and vivo, with different methods $[9,10]$ : the addition of soluble recombinant cytokines to the culture medium of CART in vitro; the systemic administration of recombinant cytokines into the patient with adoptive CD8+ T-cell therapy (ACT); the increase of cytokine receptors of CD8+ T cells: IL-7 receptor transduction contributes GD2-CAR-T the long-term and superior elimination to neuroblastoma cells, and antiapoptosis function of upregulation of bcl-2 [11]; the heterodimer cytokine and its receptor on CD8+ T cells. IL-15/IL-15R $\alpha$ IgG1-Fc heterodimer: IL-15 (IL-15N72D) binding to IL-15 receptor $\alpha$ chain and IgG Fc fusion, is almost 25 -fold effective than rhIL-15, even promotes T-cell function without lymphodepletion [12]; and IL-2-JAK-STAT3/5 pathway: a new CD19-CAR-T construct a truncated cytoplasmic domain from the IL-2 receptor $\beta$-chain (IL-2R $\beta$ ) and a STAT3binding tyrosine-X-X-glutamine (YXXQ) motif, together with the TCR signaling and CD28 domains, which owns the superior proliferative and antitumor ability via antigen-dependent activation of JAK-STAT3 $/ 5$, with the $\mathrm{CD} 4 \mathrm{RA}^{+} \mathrm{CD} 6 \mathrm{~L}^{+} \mathrm{CCR}^{+}$phenotype $[13]$.

\section{The activation of Wnt/ $\mathrm{B}$-catenin signaling}

The binding of Wnt protein to its receptors accumulates cytoplasmic $\beta$-catenin with the degradation of 'destruction complex' of the scaffolding protein APC, Axin1, CSK1 and GSK-3 $\beta$. The nucleus translocation of cytoplasmic $\beta$-catenin essentially contributes to the formation of TCF-1/LEF-1, which are critical transcriptional factors for stemness and survival of CD8+ T cells. The activation of the Wnt pathway and the blockade of GSK-3 $\beta$ (NCT01087294) both strengthen the stemness and persistence of ACT [14]. Moreover, some signaling pathways modulate the stemness of CD8 $+\mathrm{T}$ cells, maybe by controlling GSK-3 $\beta$ phosphorylation, including inhibition of PI3K/Akt signaling [15,16], and activation the notch [17]. 


\section{Blockade of immune checkpoint molecules}

The blockade protects CD8+ T cells from exhaustion. The blockade of PD-1, CTLA- 4 is proved its efficiency in mobilizing $\mathrm{T}$ cells to fight cancer cells. The FDA approved blocking antibodies targeting PD-1, CTLA-4 for melanoma, metastatic merkel cell carcinoma, kidney cancer, bladder cancer, head and neck cancers, Hodgkin lymphoma, non-small-cell lung cancer. Furthermore, the combination of exhaustion-blockade and CAR-T utilized in solid tumors (NCT03030001). Interestingly, after the PD-1 blockade, some CD8+ T cells in lymphoid tissues undergo the stem-like proliferative burst in both lymphoid and nonlymphoid tissues. TCF-1 controls the unique CD8+ T cells differentiation [18].

\section{The CD26 $6^{\text {hi }}$ T cells with stemness}

The less differentiated Tscm has stemness and it is the mainstream study for increasing persistence of antigen-specific $\mathrm{T}$ cells to enhance antitumor effect. However, a recent report from Paulos's lab showed that well-differentiated effector T cells with CD26hi phenotype can persist longer in animals and generate strong antitumor effects [19]. Thus, in addition to Tscm, the CD26hi population T cells may also be the host $\mathrm{T}$ cells to create TCR-T and CAR-T that can persist in vivo to enhance their antitumor effect.

\section{Conclusion}

The host tumor microenvironment restrains the function and survival of CD8+ T cells. The stemness of tumorspecific $\mathrm{T}$ cells gives them the longevity, proliferation and superior antitumor ability to overcome the TME obstacles. The combination strategy of the above approaches enhances the stemness induction and contributes a widespread and viable therapeutic platform in cancer immunotherapy.

\section{Financial \& competing interests' disclosure}

This work was supported by the National Natural Science Foundation of China (number: 81372781) and Provincial Natural Science Foundation of Guangdong (number: 2017A030313872). The authors have no other relevant affiliations or financial involvement with any organization or entity with a financial interest in or financial conflict with the subject matter or materials discussed in the manuscript apart from those disclosed.

No writing assistance was utilized in the production of this manuscript

\section{References}

Papers of special note have been highlighted as: $\bullet$ of interest; $\bullet \bullet$ of considerable interest

1. Gattinoni L, Klebanoff CA, Restifo NP. Paths to stemness: building the ultimate antitumour T cell. Nat. Rev. Cancer 12(10), 671-684 (2012).

-. The stem-like attributes of memory $\mathrm{T}$ cells overcome the obstacles of adoptive T-cell therapy.

2. Gattinoni L, Speiser DE, Lichterfeld M, Bonini C. T memory stem cells in health and disease. Nat. Med. 23(1), 18-27 (2017).

- The summary of Tscm function.

3. Zhang Y, Joe G, Hexner E, Zhu J, Emerson SG. Host-reactive CD8+ memory stem cells in graft-versus-host disease. Nat. Med. 11(12), 1299-1305 (2005).

4. Wu S, Zhu W, Peng Y et al. The antitumor effects of vaccine-activated CD8(+) T cells associate with weak TCR signaling and induction of stem-like memory T cells. Cancer Immunol. Res. 5(10), 908-919 (2017).

-. The weak, not strong T-cell receptor engagement signaling induces stemness of memory $\mathrm{T}$ cells.

5. Presotto D, Erdes E, Duong MN et al. Fine-tuning of optimal TCR signaling in tumor-redirected CD8 T cells by distinct TCR affinity-mediated mechanisms. Front. Immunol. 8, 1564 (2017).

6. van der Stegen SJ, Hamieh M, Sadelain M. The pharmacology of second-generation chimeric antigen receptors. Nat. Rev. Drug Discov. 14(7), 499-509 (2015).

7. Guedan S, Posey AD Jr, Shaw C et al. Enhancing CAR T cell persistence through ICOS and 4-1BB costimulation. JCI Insight 3(1), 1-17 (2018).

8. Waldmann TA, Chen J. Disorders of the JAK/STAT pathway in T cell lymphoma pathogenesis: implications for immunotherapy. Annu. Rev. Immunol. 35, 533-550 (2017).

- The IL-2/7/15 common signaling of JAK-STAT pathway controls stemness of T cells.

9. Garber K. Driving T-cell immunotherapy to solid tumors. Nat. Biotechnol. 36(3), 215-219 (2018).

10. Lamers $\mathrm{CH}$, van Steenbergen-Langeveld $\mathrm{S}$, van Brakel $\mathrm{M}$ et al. $\mathrm{T}$ cell receptor-engineered $\mathrm{T}$ cells to treat solid tumors: $\mathrm{T}$ cell processing toward optimal T cell fitness. Hum. Gene. Ther. Methods 25(6), 345-357 (2014). 
11. Johnson CB, Wrangle J, Mehrotra S et al. Harnessing the IL-7/IL-7Ralpha axis to improve tumor immunotherapy. Onco. Immunol. 5(5), e1122865 (2016).

12. Hurton LV, Singh H, Najjar AM et al. Tethered IL-15 augments antitumor activity and promotes a stem-cell memory subset in tumor-specific T cells. Proc. Natl Acad. Sci. USA 113(48), e7788-e7797 (2016).

13. Kagoya $\mathrm{Y}$, Tanaka $\mathrm{S}$, Guo $\mathrm{T}$ et al. A novel chimeric antigen receptor containing a JAK-STAT signaling domain mediates superior antitumor effects. Nat. Med. 24(3), 352-359 (2018).

-. The construction of IL-2-JAK-STAT3 signaling into the CAR-T amplified its proliferation and survival in tumor.

14. Gattinoni L, Zhong XS, Palmer DC et al. Wht signaling arrests effector T cell differentiation and generates CD8+ memory stem cells. Nat. Med. 15(7), 808-813 (2009).

15. Majchrzak K, Nelson MH, Bowers JS et al. Beta-catenin and PI3Kdelta inhibition expands precursor Th17 cells with heightened stemness and antitumor activity. JCI Insight 2(8), 1-18 (2017).

16. Abu Eid R, Ahmad S, Lin Y et al. Enhanced therapeutic efficacy and memory of tumor-specific CD8 T cells by ex vivo PI3K-delta inhibition. Cancer Res. 77(15), 4135-4145 (2017).

17. Kondo T, Morita R, Okuzono Y et al. Notch-mediated conversion of activated $\mathrm{T}$ cells into stem cell memory-like $\mathrm{T}$ cells for adoptive immunotherapy. Nat. Comm. 8, 15338 (2017).

18. Im SJ, Hashimoto M, Gerner MY et al. Defining CD8+ T cells that provide the proliferative burst after PD-1 therapy. Nature 537(7620), 417-421 (2016).

- $\quad$ PD-1 blockade bursts the proliferation of the CD8+ T cells.

19. Bailey SR, Nelson MH, Majchrzak K et al. Human CD26(high) T cells elicit tumor immunity against multiple malignancies via enhanced migration and persistence. Nat. Comm. 8(1), 1961 (2017). 